\title{
Comparing the Functional Representations of Central and Border Whiskers in Rat Primary Somatosensory Cortex
}

\author{
Barbara A. Brett-Green, Cynthia H. Chen-Bee, and Ron D. Frostig \\ Department of Neurobiology and Behavior and the Center for the Neurobiology of Learning and Memory, University of \\ California, Irvine, Irvine, California 92697-4550
}

\begin{abstract}
The anatomical representations of the large facial whiskers, termed barrels, are topographically organized and highly segregated in the posteromedial barrel subfield (PMBSF) of rat layer IV primary somatosensory cortex. Although the functional representations of single whiskers are aligned with their appropriate barrels, their areal extents are rather large, spreading outward from the appropriate barrel along the tangential plane and thereby spanning multiple neighboring and nonneighboring barrels and septal regions. To date, single-whisker functional representations have been characterized primarily for whiskers whose corresponding barrels are located centrally within the PMBSF (central whiskers). Using intrinsic signal imaging verified with post-imaging single-unit recording, we demonstrate that border whiskers, whose barrels are located at the
\end{abstract}

In the posterior medial barrel subfield (PMBSF) of rat layer IV primary somatosensory cortex (SI), a specific cell aggregate (barrel) receives thalamocortical projections from the ventroposteromedial nucleus (VPM) associated primarily with a specific whisker, whereas regions separating barrels (septa) receive thalamocortical projections primarily from the medial division of the posterior nucleus (Woolsey and Van der Loos, 1970; Killackey, 1973; Welker and Woolsey, 1974; Donaldson et al., 1975; Killackey and Leshin, 1975; Woolsey et al., 1975; Saporta and Kruger, 1977; Wise and Jones, 1978; Jensen and Killackey, 1987; Koralek et al., 1988; Lu and Lin, 1993; Land et al., 1995; Catalano et al., 1996). Cytochrome oxidase (CO) enzymatic reactivity differs between barrel and septa regions, which exhibit high versus low reactivity, respectively (Wong-Riley and Welt, 1980; Land and Simons, 1985). High CO reactivity also colocalizes with other primary sensory areas in layer IV cortex (see Fig. 1) (Wallace, 1987), with cortical regions known as perigranular or dysgranular zones (collectively referred to hereafter as DZ) exhibiting low CO reactivity (Killackey, 1973; Akers and Killackey, 1978; Chapin and Lin, 1984; Olavarria et al., 1984; Lu and Lin, 1993).

The tangential spread of the functional representation of a single whisker is rather large, spanning across multiple neighboring and non-neighboring barrels, but is still contained within the

Received June 18, 2001; revised Sept. 24, 2001; accepted Oct. 1, 2001.

This work was supported by National Institutes of Health/National Institute of Neurological Disorders and Stroke Grants NS-34519 and NS-39760 and National Science Foundation Grant IBN 9507936 (to R.D.F.). We thank Drs. M. Leon and P. Yahr for the extensive use of their cryostats, Drs. H. Killackey, R. Metherathe, D. Polley, and N. Weinberger for their helpful comments on this manuscript, and L. Banchik and E. Mendoza for assistance with the experiments.

Correspondence should be addressed to Ron D. Frostig, Department of Neurobiology and Behavior, 2205 BioSci II, University of California at Irvine, Irvine, CA 92697-4550. E-mail: rfrostig@uci.edu.

Copyright (ㄷ) 2001 Society for Neuroscience $\quad 0270-6474 / 01 / 219944-11 \$ 15.00 / 0$ borders of the PMBSF, also evoke large activity areas that are similar in size to those of central whiskers but spread beyond the PMBSF and sometimes beyond primary somatosensory cortex into the neighboring dysgranular zones. This study indicates that the large functional representation of a single whisker is a basic functional feature of the rat whisker-to-barrel system and, combined with results from other studies, suggest that a large functional representation of a small, point-like area on the sensory epithelium may be a functional feature of primary sensory cortex in general.

Key words: central whisker; border whisker; whisker functional representation; intrinsic signal imaging; rat barrel cortex; dysgranular zone columnar border of layer IV PMBSF (Orbach et al., 1985; Grinvald et al., 1986; Armstrong-James and Fox, 1987; ArmstrongJames et al., 1991, 1992; Narayan et al., 1994; Chen-Bee and Frostig, 1996; Kleinfeld and Delaney, 1996; Masino and Frostig, 1996; Prakash et al., 1996; Hodge et al., 1997; Peterson et al., 1998; Sheth et al., 1998; Ghazanfar and Nicolelis, 1999; Polley et al., 1999a,b). Intracortical projections are thought to provide one anatomical substrate for this large activity spread (Akers and Killackey, 1978; Chapin et al., 1987; Koralek et al., 1990; Armstrong-James et al., 1991; Fabri and Burton, 1991; Fox, 1994; Hoeflinger et al., 1995; Gottlieb and Keller, 1997; Schroder and Luhmann, 1997; Goldreich et al., 1999; Kim and Ebner, 1999). Because research has been focused mainly on whiskers whose barrels are located centrally within the PMBSF (central whiskers), it is unclear whether a whisker with a barrel at the border of the PMBSF (border whisker) also has a large functional representation. Although whisker responses have been recorded up to $\sim 300 \mu \mathrm{m}$ away from the appropriate barrel into the DZ immediately surrounding the PMBSF (Chapin and Lin, 1984), to date, activity evoked by border whiskers in this DZ has not been characterized. The current study seeks to determine whether neighboring DZ confines the tangential spread of whisker-evoked activity to the columnar border of the PMBSF or whether the functional representation of a border whisker can extend to far distances beyond the PMBSF (and thus beyond SI for some border whiskers; see Fig. 1) into adjacent DZ and perhaps into other neighboring primary sensory areas not known to process whisker information.

Using intrinsic signal optical imaging (ISI) (Grinvald et al., 1986; Frostig et al., 1990; Ts'o et al., 1990), we characterized the functional representation of two border whiskers, A2 and E2, as well as a central whisker, C2 (see Fig. 1). The A2 barrel is situated 
at the posterolateral border of the PMBSF, and hence SI, near the border of presumed secondary somatosensory cortex (Welker and Sinha, 1972; Carvell and Simons, 1987; Wallace, 1987; Koralek et al., 1990; Fabri and Burton, 1991) and primary auditory cortex (AI). The E2 barrel is located at the opposite PMBSF border near the anteromedial DZ closest to the border of the trunkforepaw region of layer IV SI. We chose two whiskers at opposite PMBSF borders to compare the tangential spread of evoked activity toward a primary sensory area of a different modality (i.e., AI) versus nonwhisker regions within SI. Post-imaging single-unit recordings were performed in a subset of animals to compare optical versus spiking activity evoked by identical whisker stimulation. Our results demonstrate that the functional representation of border whiskers is similarly large to that of central whiskers, spreading tangentially beyond the PMBSF and in some cases beyond the SI.

\section{MATERIALS AND METHODS}

Subjects. Thirty-six adult male Sprague Dawley rats (Charles River Laboratories, Wilmington, MA) were briefly anesthetized with the inhalant halothane before receiving an intraperitoneal injection of Nembutal $(50 \mathrm{mg} / \mathrm{kg})$. Supplemental intraperitoneal injections of Nembutal $(10-15 \mathrm{mg} / \mathrm{kg})$ were delivered during the course of each experiment as necessary to maintain mild withdrawal and corneal reflexes. Heart rate and rectal temperature were continuously monitored (model 78354A; Hewlett-Packard, Palo Alto, CA), and an adjustable heating pad was used to maintain body temperature at $\sim 37^{\circ} \mathrm{C}$.

Animal preparation and whisker stimulation. For a more detailed description, see Masino et al. (1993). A large rectangular area $\left(\sim 40 \mathrm{~mm}^{2}\right)$ of exposed skull to the left of the central suture was thinned to a thickness of $\sim 150 \mu \mathrm{m}$. A Vaseline well was built around the border of the thinned skull area, filled with silicon oil (200 fluid; viscosity, $50 \mathrm{cs}$; Accumetric, Elizabethtown, KY), and capped with a glass coverslip, making the skull transparent and the underlying vasculature easily discernable. A single whisker (A2, C2, or E2) of the right mystacial pad was deflected with a computer-controlled pulse generator (Master 8; A.M.P.I.) wire probe system (Bakin Systems 2, Irvine, CA) for $1 \mathrm{sec}$ at 5 $\mathrm{Hz}$ in the rostral-to-caudal direction (amplitude and duration of one deflection were $1.9^{\circ}$ and $100 \mathrm{msec}$, respectively). Although sometimes used to prevent inadvertent stimulus delivery, the trimming of nonstimulated whiskers may affect cortical responses and thus was not performed in the present study. However, microscopic inspection of the mystacial pad revealed that this stimulation left neighboring whiskers as well as the fur on the snout undisturbed. Also, delivery of whisker stimulus did not emit any discernable auditory cues, and blocks of imaging and single-unit control trials collected between stimulation trials revealed a lack of evoked activity when the wire probe was repositioned such that it no longer deflected any whiskers.

Intrinsic signal optical imaging. All rats were placed under the camera such that the horizontal axis of the imaging data were parallel to the rostrocaudal neuroaxis. Because the thinned skull area was located above a region of the brain that exhibits some degree of curvature, the head of the animal was then rotated along the midline axis so that the optical axis of the imaging camera lens was perpendicular to the central region of the thinned skull surface. A slow-scan charge-coupled device (CCD) camera (PhotoMetrics, Tucson, AZ) equipped with an inverted $50 \mathrm{~mm} \mathrm{AF}$ Nikon (Tokyo, Japan) lens (Nikon 1:1.8) combined with an extender (model PK-13; Nikon) was then positioned above the Vaseline well, and an image of the surface vasculature was taken to serve as a reference for subsequent procedures. Data were collected from a $6.8 \times 5.1 \mathrm{~mm}$ area of thinned skull and represented with a $192 \times 144$ pixel array. Therefore, each pixel represented a $35 \times 35 \mu \mathrm{m}$ area of cortex. The CCD camera was defocused $300 \mu \mathrm{m}$ below the cortical surface before imaging. Two fiber-optic light guides driven by a stabilized power supply (ATE 15$15 \mathrm{M}$; Kepco Inc., Flushing, NY) continuously illuminated the cortex with light that passed through a $630 \mathrm{~nm}$ red light filter (bandpass, $30 \mathrm{~nm}$; Omega Optical, Brattleboro, VT). Light reflectance values were collected by the CCD camera in $500 \mathrm{msec}$ frames. Trials lasted $4.5 \mathrm{sec}$ each, containing nine $500 \mathrm{msec}$ frames. Stimulation trials began with $1 \mathrm{sec}$ of baseline activity, followed by $1 \mathrm{sec}$ of activity collected during whisker stimulation and $2.5 \mathrm{sec}$ of activity collected after stimulus offset, with an intertrial interval of $15 \mathrm{sec}$. A complete data session consisted of 128 trials.

Imaging data were quantified using a modified protocol described in detail previously (Chen-Bee et al., 2000). For each pixel within the $192 \times$ 144 pixel array, a ratio value was created with an intratrial division analysis such that the average IS activity occurring 0.5 up to $1.5 \mathrm{sec}$ poststimulus onset was divided by $500 \mathrm{msec}$ of prestimulus IS activity occurring immediately before stimulus onset. After the ratio values were processed with a Gaussian filter (half-width of 5), the areal extent of the functional representation was quantified by thresholding at three levels above prestimulus activity collected during the $1 \mathrm{sec}$ immediately preceding stimulus onset (listed in order of increasing signal strength): 1.5 , 2.0 , and $2.5 \times 10^{-4}$ increments. These levels were chosen based on previous characterization of the range of ratio values typically found in data sessions that contained whisker-evoked activity. Statistical comparisons of areas quantified at all three thresholds of evoked activity were conducted with a repeated-measures ANOVA (Systat 7; SPSS, Chicago, IL). Images of evoked activity were generated by applying an eight-bit, linear grayscale map to the unfiltered ratio values so that the area of evoked activity as quantified with the $2.0 \times 10^{-4}$ threshold could be visualized as a black patch.

In addition, for every functional representation, we also determined whether it was anisotropic and, if so, what was the orientation of the anisotropic representation. We quantified the degree of anisotropy by first measuring the length of the largest (major axis) and smallest (minor axis) diameter of the functional representation and then calculating the ratio between the major and minor axes. Because the areal extent of each functional representation was quantified at three thresholds, three measures of anisotropy were obtained per functional representation. In cases in which a functional representation was deemed anisotropic, its orientation was determined by measuring the angle between the major axis of that functional representation and the horizontal axis. Because the rats were identically positioned under the camera before imaging, the horizontal axis of all imaging data were equivalent to the rostrocaudal neuroaxis. Up to three orientations may be obtained per functional representation if, after areal extent quantification, the representation was deemed anisotropic at all three thresholds.

Lesioning and $\mathrm{CO}$ histochemistry. After imaging, the cortex was lesioned $(10 \mu \mathrm{A}, 10 \mathrm{sec})$ and then processed for $\mathrm{CO}$ histochemistry. The outer limits of the imaged area were marked on an image of the vasculature taken at the beginning of each experiment, and lesions were made in layer IV at these locations so that the orientation of the imaged area could be delineated relative to the $\mathrm{CO}$ staining pattern. In addition, lesions were made at the locations of peak IS response obtained by stimulating separately single whiskers A2, C2, or E2. To be processed for $\mathrm{CO}$, animals were given a lethal injection of Nembutal and perfused intracardially with $0.1 \mathrm{M}$ sodium phosphate buffer, $\mathrm{pH} 7.4$, followed by $4 \%$ paraformaldehyde. The brain was then removed, and the left cortex was dissected and flattened between two glass slides to a thickness of $\sim 2$ $\mathrm{mm}$. The tissue was cryoprotected in $30 \%$ sucrose and sectioned tangential to the pial surface at $40-50 \mu \mathrm{m}$. Sections were processed according to the protocol described by Wong-Riley and Welt (1980), mounted on gelatin-coated slides, air-dried, and coverslipped with Permount. In six animals, imaging was not followed by lesioning, and therefore the areas of their A2, C2, and E2 barrels were quantified for statistical comparisons using repeated-measures ANOVA (Systat 7; SPSS). The CO staining pattern from one of these animals is provided in the left panel of Figure 1.

Single-unit recording. Imaging was followed by single-unit recording in five animals ( $n=4$ for whisker A2 stimulus; $n=1$ for whisker E2 stimulus). Accordingly, a craniotomy was performed inside the border of the thinned skull area, and a portion of the dura was retracted. The Vaseline well was rebuilt surrounding the exposed area and filled with silicon oil to keep the cortex moist. Tungsten microelectrodes (impedance, $\sim 1.0 \mathrm{M} \Omega$; Micro Probe Inc., Potomac, MD) were advanced normal to the pia with a hydraulic microdrive (David Kopf Instruments, Tujunga, CA) to record whisker-evoked single-unit activity. Stimulation parameters were identical to those used during imaging. Stimulation trials included $1 \mathrm{sec}$ of baseline activity, followed by $1 \mathrm{sec}$ of activity collected during whisker stimulation and $1 \mathrm{sec}$ of activity collected after stimulus offset. A complete data session consisted of 100 stimulation trials, with an intertrial interval of $10 \mathrm{sec}$. For each animal in which single-unit recordings were performed, three penetrations were made. The first penetration was invariably made at the location of the peak IS response. The second and third penetrations were made at increasing 

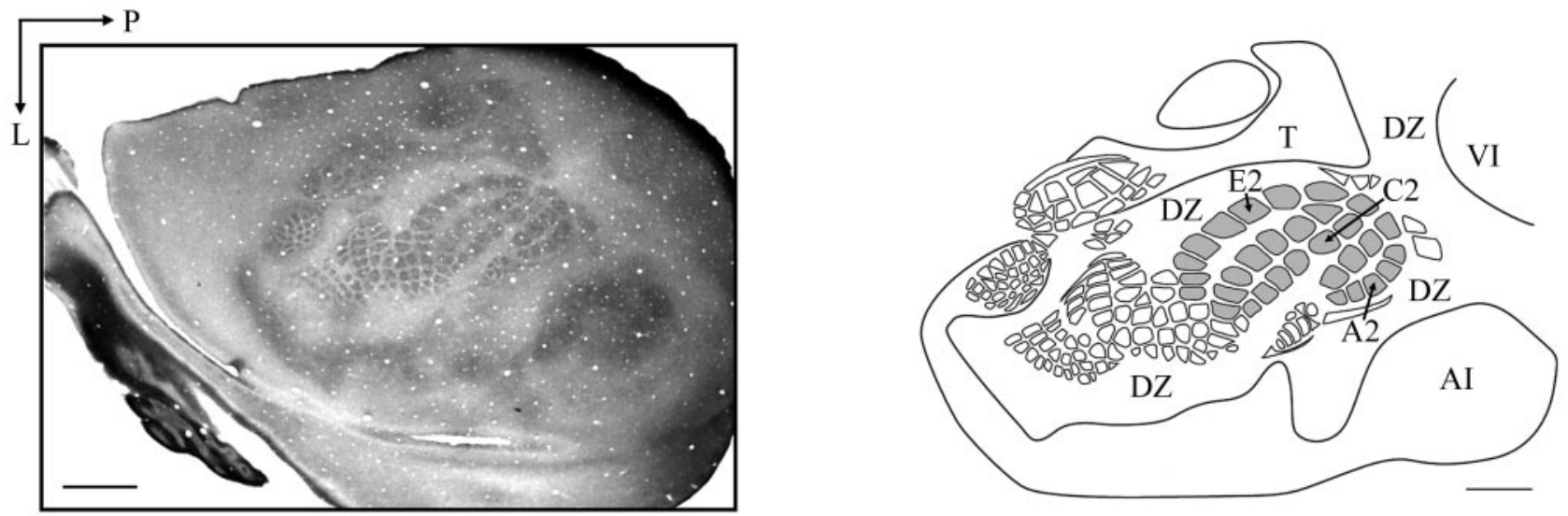

Figure 1. Layer IV CO map. Left, Photomicrograph of a $40 \mu \mathrm{m}$ tangential section of the rat left cortex through layer IV that has been stained for CO. Dark patches correspond to areas of high CO reactivity. Right, Template of the layer IV CO map created from the tangential section presented in $A$, with the PMBSF shaded in gray. Regions of high CO reactivity that receive V PM projections corresponding to whisker A2, C2, or E2 are labeled, respectively. $T$, Trunk; VI, primary visual cortex. SII is located posterolaterally to the PMBSF (data not shown). Orientation applies to both panels; $L$, lateral; $P$, posterior. Scale bars, $1 \mathrm{~mm}$.

distances from the first penetration. Recordings were made in both the supragranular $(300-400 \mu \mathrm{m})$ and granular $(600-700 \mu \mathrm{m})$ layers in each penetration. A waveform discriminator (MultiSpike Detector; Yissum, Jerusalem, Israel) allowed up to three different neurons to be simultaneously isolated per recording location. After completion of all recordings, electrolytic lesions (5 $\mu \mathrm{A}, 10 \mathrm{sec}$; Iso-Flex stimulator; A.M.P.I.) were made in layer IV for histological verification of recording locations relative to the $\mathrm{CO}$ map.

Peristimulus time histograms (PSTHs) consisting of 100 trials and a bin width of $1 \mathrm{msec}$ were generated for each neuron recorded using HIST software (Spike Systems). Significance of excitatory "on" responses $(p<$ 0.01) was evaluated relative to prestimulus spontaneous activity (Abeles, 1982). Latency and magnitude of the first significant response were measured for each PSTH. Latency was measured by identifying the first $1 \mathrm{msec}$ bin that contained a significant response after stimulus onset. Response magnitude of that same bin was measured by subtracting the mean prestimulus spontaneous firing rate from its firing rate. An average of each of these two measures was obtained for up to three neurons recorded simultaneously from the same electrode at the same depth (either supragranular or granular) in a penetration. Subsequently, the data were grouped according to the distance of a penetration from the peak of IS response, and latency and magnitude averages were obtained across animals.

Alignment between imaging data and underlying $C O$ maps. The alignment procedure was performed in the four animals (whisker A2 stimulus) in which the location of electrodes inserted normal to the cortical surface were verified with electrolytic lesions in layer IV. In each animal, after finalizing the position of the CCD camera, an image of the surface vasculature was taken to serve as reference and imported into Photoshop (version 6.0; Adobe Systems, San Jose, CA). Marks were placed on this image to indicate the location of the perpendicular electrode insertions into the cortex (three insertion locations per animal). Photographs of the CO-stained tangential cortical sections (40-50 $\mu \mathrm{m}$ in thickness) were also imported into Photoshop for each animal. Two tangential sections were needed per animal to generate a composite $\mathrm{CO}$ map that best captured the electrolytic lesions as well as key cortical regions, such as the PMBSF (particularly the posterolateral border), portions of AI and VI nearest the PMBSF, and the DZ separating these primary sensory regions. For each animal, the image of its surface vasculature was then superimposed over the appropriate composite CO map using Photoshop and then (1) rescaled to that of the $\mathrm{CO}$ map, (2) moved such that the first insertion site (corresponding to recording in and above the A2 barrel) was located above the electrolytic lesion in the A2 barrel, and (3) rotated until the axis formed by the three insertion sites matched the axis formed by the three electrolytic lesions. Last, for each animal, the analysis results of imaging data underwent the same rescaling, movement, and rotation steps determined for that animal before being spatially compared with the appropriate $\mathrm{CO}$ map.

\section{RESULTS}

\section{The functional representations of central and border whiskers are similarly large}

Data from representative cases for the central whisker $\mathrm{C} 2$ and the border whiskers A2 and E2 are provided in Figure 2. As expected, we found that the functional representation of a centrally located whisker is large (Fig. 2, middle row) and that response magnitude decays progressively over a distance of $>1 \mathrm{~mm}$ from peak activity, with peak activity located above the appropriate barrel as reported previously (Chen-Bee and Frostig, 1996; Masino and Frostig, 1996; Prakash et al., 1996; Polley et al., 1999a,b). Similar findings were obtained for whisker A2, whose barrel is located at the posterolateral border of the PMBSF, in that the peak activity is also located above the appropriate barrel and a single, large area of evoked activity is also observed (Fig. 2, top row). The large area of evoked activity observed for the border whisker A2 could potentially be attributable to evoked activity in the secondary somatosensory region (SII) because of its proximity to the whisker A2 barrel: SII is located posterolateral to the PMBSF (Welker and Sinha, 1972; Carvell and Simons, 1987; Wallace, 1987; Koralek et al., 1990; Fabri and Burton, 1991) and is partially overlapping with the posterolateral DZ. However, this is unlikely for several reasons. First, the spatial spread of activity evoked by stimulation of whisker A2 was not comprised of two distinct activity areas abutting each other but instead consisted of a single activity area, with the strength of activity decreasing smoothly away from the peak activity without any obvious transition.

Furthermore, whisker E2, whose barrel is located at the anteromedial border of the PMBSF, and whisker C2, whose barrel is centrally located, also evoked a single, large area of activity, with the activity strength decaying smoothly away from the peak activity (Fig. 2, bottom and middle rows), further supporting the likelihood that stimulation of only a single whisker may not be sufficient to evoke activity in SII as assessed with intrinsic signal imaging. Similar spatiotemporal characteristics were also found after the imaging of the functional representations of other single border whiskers $(\alpha, \beta$, and $\mathrm{SO})$, thus generalizing $\mathrm{E} 2$ and $\mathrm{A} 2$ findings to other border whiskers (data not shown).

The size of single-whisker functional representations was 

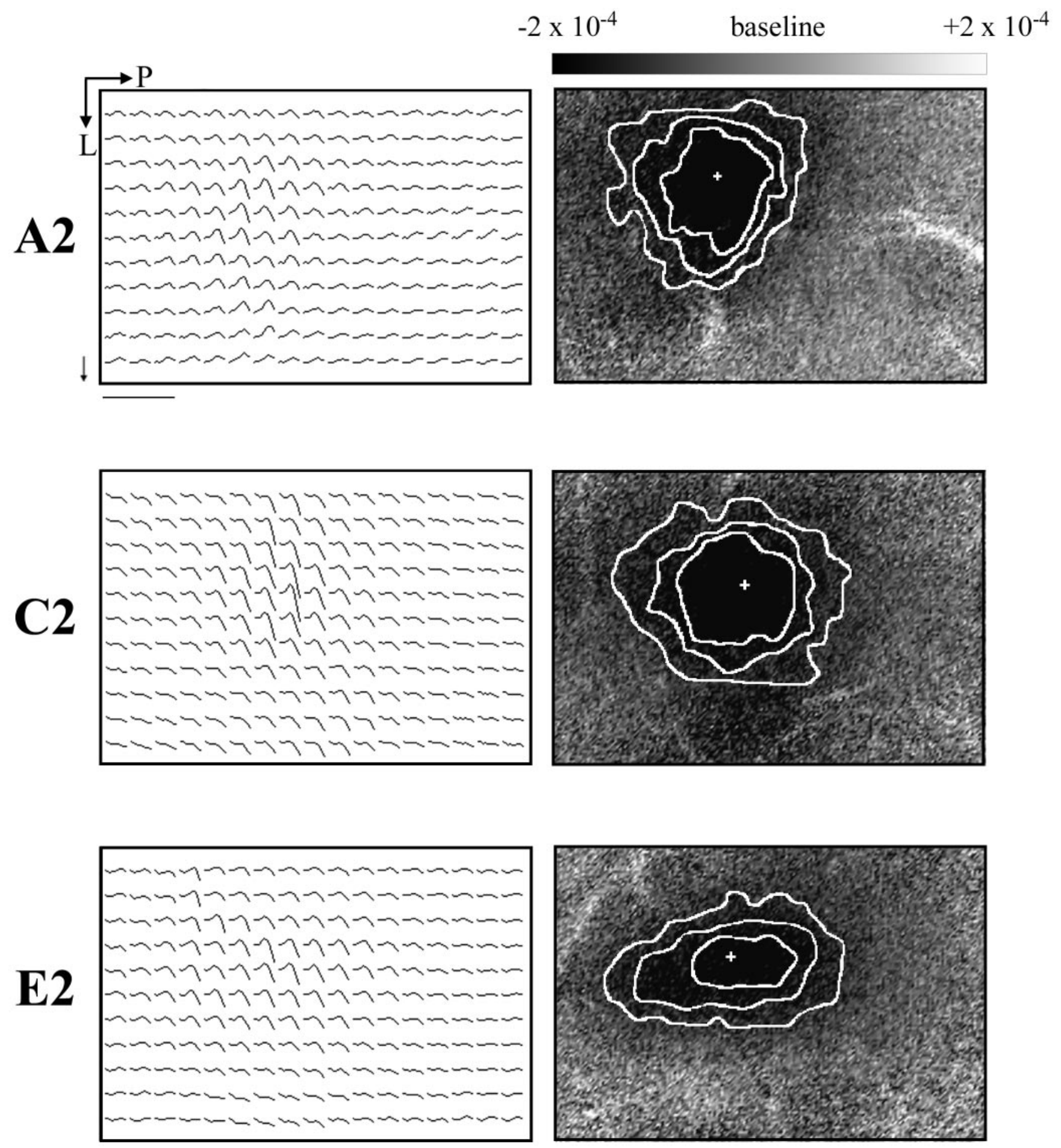

Figure 2. Stimulation of a single central or border whisker evokes a similarly large area of activity. Left column, Spatiotemporal arrays of intrinsic signals evoked by stimulating whisker A2 (top row), C2 (middle row), or E2 (bottom row) in three representative animals are provided. Each trace represents the average intrinsic signal for the underlying $0.35 \times 0.35 \mathrm{~mm}$ cortical region $(10 \times 10$ pixels $)$ and depicts whisker-evoked changes in light reflectance over $4.5 \mathrm{sec}$. One second of stimulation is delivered to one whisker after $1 \mathrm{sec}$ of prestimulus activity is collected. By convention, the traces are plotted as upgoing, although cortical activation causes a decrease in light reflectance. Vertical scale bar shown as an arrow: $1 \times 10^{-3}$ fractional change; applies to all three left panels. Scale bar, $1 \mathrm{~mm}$. Orientation applies to all six panels; $L$, lateral; $P$, posterior. Right column, After data processing, the size of the evoked activity area is quantified using three arbitrary threshold levels away from baseline. The areas quantified with the use of the $1.5,2.0$, and $2.5 \times$ $10^{-4}$ threshold levels correspond to the outermost, middle, and innermost white outlines, respectively. An eight-bit, linear grayscale map is applied to the processed data so that the cortical region exhibiting evoked activity of at least $-2.0 \times 10^{-4}$ away from baseline can be visualized as a black patch Location of peak activity is indicated with a + symbol. 
Figure 3. The size of the functional representation of a whisker is similar between whiskers A2, C2, and E2. $A$, The area of the functional representation of a whisker as quantified using three threshold levels above baseline is plotted here for 21 rats $(n=7$ rats each for whisker A2, C2, and E2). Note the large size of single-whisker functional representations on the order of several squared millimeters. $B$, The quantified area values after transformation with the square root function. C, Top, Scatterplot of means versus SEs calculated for each subgroup of area values after subdividing the data by whisker type and threshold type. Note the large range of SEs across means, as well as the positive relationship between SEs and means. Bottom, Scatterplot of means versus SEs calculated for each subgroup of transformed area values after subdividing the data by whisker type and threshold type. Note the substantial reduction in the range of SEs across means and the removal of the positive relationship between SEs and means. $D$, Bar graphs with SEs are provided for the transformed values of the quantified areas of single-whisker functional representations. Although there was a tendency for whisker A2 functional representations to be smaller, a repeatedmeasures ANOVA on the transformed data found no significant difference between the three whiskers across all thresholds $\left(F_{(2,18)}=1.94 ; p=0.17\right)$. All nine possible pairwise comparisons using separate variance unpaired $t$ tests also found no significant difference between any two combinations of whiskers for any threshold ( $p>0.05$ for all comparisons).
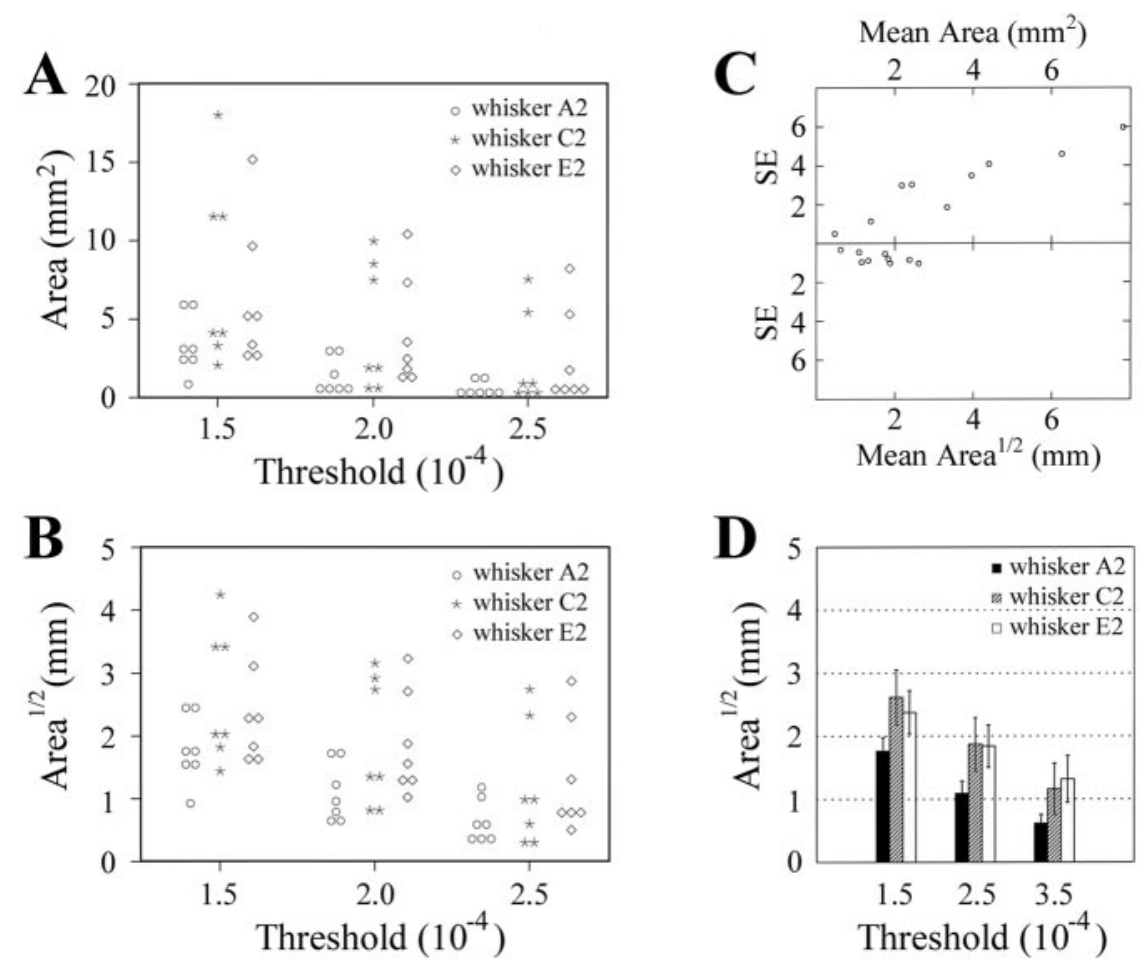

quantified at three threshold levels of activity for 21 rats (A2, $n=$ 7; C2, $n=7$; and E2, $n=7$ ) (Fig. $3 A$ ). After grouping the area values by whisker type and threshold type, means and SEs were calculated for each of the nine subgroups (three whiskers by three activity thresholds). As illustrated in the top half of Figure $3 C$, the $\mathrm{SE}$ of each mean ranged between 0.50 and $5.96 \mathrm{~mm}^{2}$, and the SE increased with increasing mean. Both observations violated two conditions required by a repeated-measures ANOVA: (1) homogeneity of variance across means; and (2) independence between variance and mean. The transformation of the area data with the square root function (Fig. $3 B$ ) resolved the above two violations, as evidenced by the substantial reduction in the range of SEs across means $(0.34-1.05 \mathrm{~mm})$ and the removal of the positive relationship between SEs and means (Fig. 3C, bottom). Thus, statistical analysis was conducted on the transformed data (Fig. $3 D)$. Although there was a tendency for whisker A2 functional representations to be smaller (Fig. 3), we found no significant difference in the size of the functional representation between the three whiskers across all activity thresholds $\left(F_{(2,18)}=1.94\right.$; $p=0.17$; repeated-measures ANOVA). This lack of significant difference between whiskers was also obtained with all nine possible pairwise comparisons ( $p>0.05$ for all comparisons) using separate variance unpaired $t$ tests, which do not require homogeneity of variance or independence between SEs and means.

As illustrated in Figure 2, the functional representation of a single whisker can be anisotropic. It has been shown previously that the whisker barrels of the PMBSF are organized into rows and arcs such that, within the same row, more caudal barrels are more medial compared with more rostral barrels (Fig. 1) (for rat PMBSF, see Welker and Woolsey, 1974; Killackey et al., 1976; Land and Simons, 1985). Furthermore, stimulation of a single whisker appears to evoke an anisotropic spread of single-unit activity in the PMBSF that tends to orient along a row of barrels (Chapin 1986; Armstrong-James et al., 1992). To determine whether there is a systematic anisotropy in the functional representation of whisker A2, C2, and E2 as characterized in the present study, we calculated the major-to-minor ratio for each functional representation quantified at each of three thresholds, in which a value of 1 indicated a lack of anisotropy, and increasing values indicated increasing degrees of anisotropy. To identify the criterion value in which to qualify a representation as being anisotropic, we used stem-and-leaf plots of major-to-minor ratio values to identify the first obvious break in the distribution of values as shared across all three whiskers and all three thresholds. This obvious break led to two subdistributions: $1.04-1.21$ versus 1.27-3.12. These two subdistributions successfully divided the functional representations into those with negligible anisotropy and those with apparent anisotropy as verified by the overall shape of the functional representations.

Collectively across all three whiskers and all three thresholds, anisotropy was deemed negligible in $38 \%$ of the functional representations and thus were considered not to favor a particular orientation. For those cases in which a functional representation was deemed anisotropic, the orientation for that functional representation was determined as follows: (1) $0^{\circ}$ indicated that its major axis was parallel to the rostrocaudal axis; (2) either a positive or negative angle of $90^{\circ}$ indicated that its major axis was perpendicular to the rostrocaudal axis; (3) positive angles between $0^{\circ}$ and $90^{\circ}$ indicated that the caudal portion of the functional representation was more medial than the rostral portion; and (4) negative angles between $0^{\circ}$ and $90^{\circ}$ indicated that the rostral portion was more medial. Therefore, an anisotropic functional representation with a positive angle between $0^{\circ}$ and $90^{\circ}$ would have its major axis oriented more along a row of barrels. For those functional representations deemed to be anisotropic, the orientation was observed to range between $-68.2^{\circ}$ and $+86.2^{\circ}$ and appeared to exhibit no systematic trend for any of the three whiskers; for each whisker, (1) the distribution of orientations was observed to contain both negative and positive values, (2) 

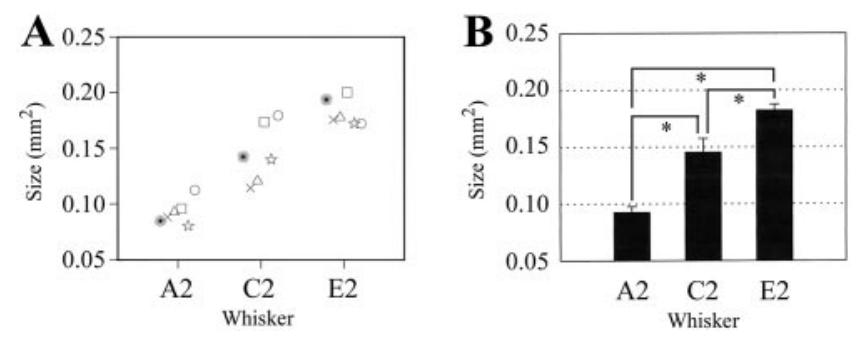

Figure 4. Significant differences in barrel size between whiskers A2, C2, and $\mathrm{E} 2 . A$, The quantified barrel size of whiskers $\mathrm{A} 2, \mathrm{C} 2$, and $\mathrm{E} 2$ is plotted here for six rats. Note the similar spread in area values between the different whiskers, which satisfies the homogeneity of variance requirement of a repeated-measures ANOVA. $B$, Means and SEs are provided for the size of A2, C2, and E2 barrels. A repeated-measures ANOVA found a significant difference in barrel size between the three whiskers $\left(F_{(2,10)}=51.91 ; p=0.00001\right)$, with the A2 barrel smaller than the $\mathrm{C} 2$ barrel, which in turn was smaller than the E2 barrel. ${ }^{*} p<0.05$, significant differences between barrel sizes as tested with contrasts.

there were instances in which within the same functional representation anisotropy was deemed negligible at one threshold and apparent at another threshold, and (3) there were instances in which the same functional representation was deemed anisotropic across multiple thresholds but exhibited extreme differences in the orientation (i.e., a positive angle in one case and a negative angle in the other case). In all examples of anisotropy, the surface vasculature was observed to contribute in some way to the orientation of the functional representation. Whether our results also correlated with the orientation of the PMBSF (e.g., the major axis of a functional representation may potentially orient more along a row of barrels) is a possibility in need of additional research and is beyond the scope of the present study, although this possibility is unlikely given the lack of a systematic orientation for any of the functional representations investigated in the present study, which is in contrast to the known orientation of the PMBSF in which more caudal barrels within the same row are more medial compared with more rostral barrels.

In six additional animals, the size of the A2, C2, and E2 barrels was quantified for comparison (Fig. $4 A$ ). Because the data set of barrel sizes satisfied the assumptions of a repeated-measures ANOVA, there was no need to transform the data before statistical analysis. In contrast to their similarly sized functional representations, we found a significant difference in barrel size between whiskers A2, C2, and E2 $\left(F_{(2,10)}=51.91 ; p=0.00001\right.$; repeated-measures ANOVA), with the A2 barrel smaller than the C2 barrel, which, in turn, was smaller than the E2 barrel (for details, see Fig. $4 B$ ). It should be noted that, even at the highest threshold criterion $\left(2.5 \times 10^{-4}\right)$, the average size of the functional representation of a single whisker (Fig. 3A) was 5-15 times larger than the average size of the appropriate barrel (Fig. 4B).

\section{Evoked and single-unit responses are found beyond the PMBSF}

In four animals, imaging of the whisker A2 functional representation was followed by single-unit recording from both the supragranular and granular layers at three cortical locations (Figs. 5, 6) to determine the extent to which the imaging data had underlying evoked spiking activity from single units. For each animal, the location of the electrodes when they first penetrate the cortex was marked on an image of the cortical surface for future reference. The first penetration was perpendicular to the cortical surface at a location closest to the peak IS response without puncturing surface blood vessels. The second and third penetrations were also perpendicular to the cortical surface at increasing distances from the first penetration in the posterolateral direction (Fig. $5 A$ ). Electrolytic lesions made subsequent to single-unit recording confirmed that the first electrode penetration was aligned with the A2 barrel in each animal (Fig. 5B). The second and third electrode penetrations were located at increasing distances away from the peak activity, averaging 0.57 and $1.05 \mathrm{~mm}$ away from the center of the A2 barrel along the tangential plane, respectively, with the border of AI located at an average distance of $1.26 \mathrm{~mm}$.

We were interested in determining whether the large spread of the whisker A2 functional representation extended beyond the PMBSF along the tangential plane and thus intended to superimpose the imaging data on top of the appropriate $\mathrm{CO}$ section. However, despite our efforts to ensure that the optical axis of the imaging camera was perpendicular to the central region of the imaged cortex, the total region of the imaged cortex was rather large and therefore expected to exhibit some curvature, especially toward the periphery. Thus, we felt it necessary to first characterize the spatial relationship between the imaging data collected from a curved surface view versus the flat $\mathrm{CO}$ map of the PMBSF. An example of the procedure is provided in Figure 5. By aligning the locations of the electrode penetrations that were marked on an image of the cortical surface (Fig. $5 A$ ) with their corresponding electrolytic lesions in layer IV (Fig. $5 B$ ), we verified that a good spatial correspondence existed between the central region of the imaged cortical surface, extending $\sim 1 \mathrm{~mm}$ away from the peak activity in the posterolateral direction, and the CO-stained tangential section through the PMBSF (Fig. 5C).

After rotating the imaging data according to the alignment procedure described above, we superimposed the rotated imaging data over the appropriate $\mathrm{CO}$ section (Fig. $5 D$ ). We found that the functional representation of whisker A2 spread beyond the PMBSF along the tangential plane into the DZ separating the PMBSF and AI. Data from a representative animal is provided in Figure 6, illustrating the following correspondence between intrinsic signal and single-unit activity: (1) the location of the strongest single-unit activity coincided with the location of the strongest intrinsic signal activity, with both aligned with the A2 barrel; and (2) both single-unit and intrinsic signal activity decayed in strength with increasing distance from the A2 barrel along the tangential plane. A summary of results averaged across four animals is provided in Table 1, demonstrating that singleunit responses were evoked beyond the PMBSF in all four animals at an average distance of $570 \mu \mathrm{m}$ away from the center of the A2 barrel along the tangential plane (i.e., within the DZ separating the PMBSF and AI) in both the supragranular and granular layers. In three of four animals, no single-unit responses were obtained at the furthest electrode recording location. In two of these three animals, the furthest recording location was just outside the border of AI, whereas for the third animal, we could not determine the recording location relative to the AI border. In the fourth animal, evoked single-unit responses were obtained at the furthest penetration located $1.26 \mathrm{~mm}$ away from the center of the A2 barrel along the tangential plane (Fig. 6). For this fourth animal, although the farthest recording location appeared just inside the AI border, the determination of the AI border was somewhat ambiguous (Fig. 6). Thus, in all four animals, whiskerevoked intrinsic signal and single-unit activity was observed in the DZ separating the PMBSF and AI, but the issue of whether whisker-evoked activity extended into AI still remains unresolved. For the single units that were significantly evoked by 
Figure 5. Spatial correspondence between the imaging data collected from a curved surface view versus a flat CO map of the PMBSF. $A$, The functional representation of whisker A2 as quantified at three increasing activity thresholds (black outlines) are superimposed on an image of the blood vessel pattern overlying the curved cortical surface as viewed through the thinned skull by the imaging camera. Also superimposed are the location of peak activity (black cross) and the location of the three electrode penetrations at the point of insertion into the cortical surface (white circles). Scale bar, $1 \mathrm{~mm}$. Orientation applies to all panels; $A$, anterior; $L$, lateral. $B$, Electrolytic lesions in layer IV of the cortex stained for $\mathrm{CO}$ confirmed that the first electrode penetration was aligned with the A2 barrel, whereas the second and third penetrations were located at increasing distances away from the A2 barrel in the posterolateral direction toward primary auditory cortex (black arrows). C, After rotating the locations of the electrode penetrations at the cortical surface and positioning the first penetration above the A2 barrel, a good spatial correspondence was found between the locations of the two remaining electrode penetrations at the cortical surface (black circles) and their corresponding electrolytic lesions in layer IV of the cortex, despite the surface curvature of the intact cortex and the shrinkage of cortical tissue after fixation. $D$, After rotating the imaging data using the same alignment proce-
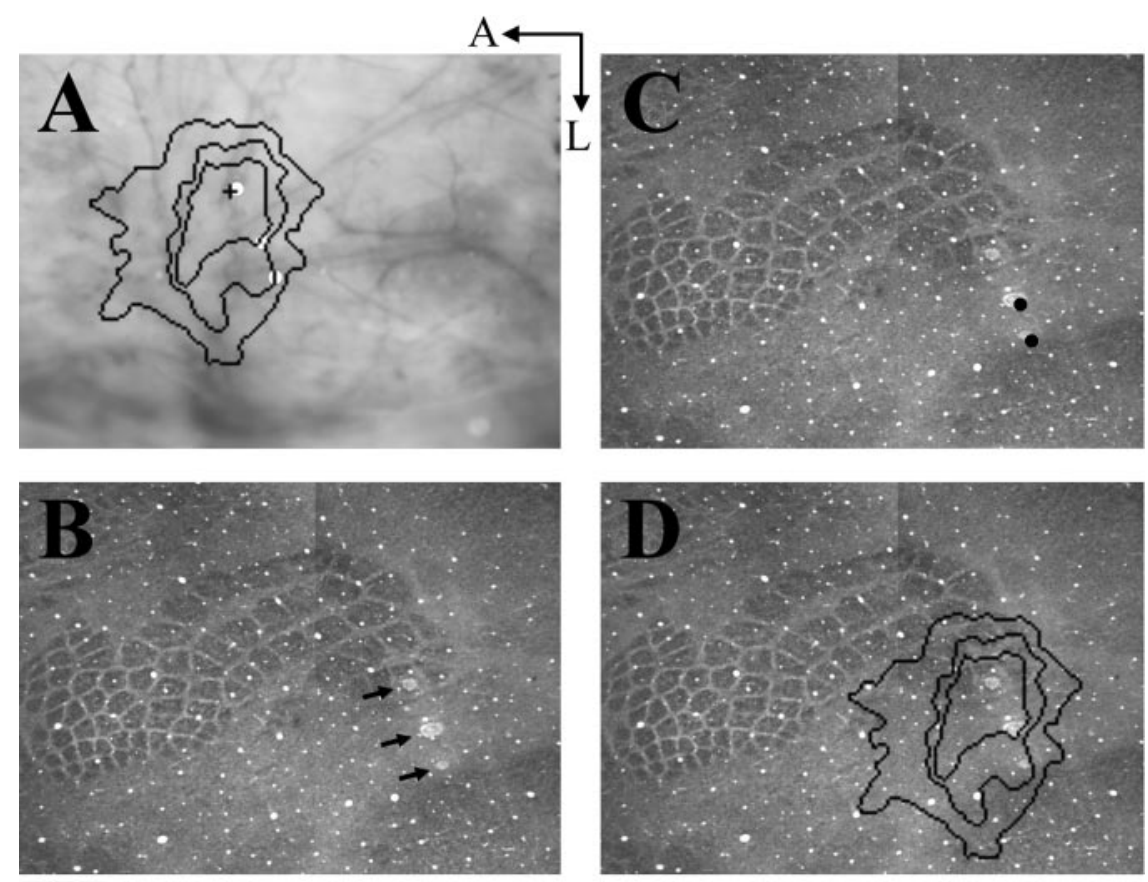

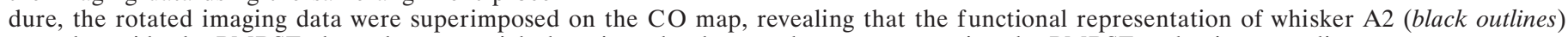
spread outside the PMBSF along the tangential plane into the dysgranular zone separating the PMBSF and primary auditory cortex.

whisker A2 stimulation, the shortest mean latency was observed within the A2 barrel (mean $\pm \mathrm{SE} ; 7.95 \pm 0.38 \mathrm{msec}$ ). Mean latencies were observed to increase with increasing distance from the A2 barrel for both the supragranular (from $11.25 \pm 0.55$ to $30.33 \pm \mathrm{NA}$ ) and granular (from $7.95 \pm 0.38$ to $34.00 \pm \mathrm{NA}$ ) layers. Thus, as with findings for single-unit responses evoked by stimulation of a centrally located whisker (Chapin, 1986; Armstrong-James and Fox, 1987; Armstrong-James et al., 1991; Fox, 1992, 1994; Simons et al., 1992; Ghazanfar and Nicolelis, 1999; Polley et al., 1999a; Petersen and Diamond, 2000), we obtained results for the border whisker A2 in which, on average, evoked single-unit responses exhibited longer latencies and weaker magnitudes with increasing distance from the appropriate barrel within both the supragranular and granular layers. Because our primary purpose for performing single-unit recordings was to verify the presence of spiking neurons evoked by whisker A2 stimulation, a verification that was particularly necessary in the DZ because this region was not necessarily thought to process whisker information, we did not investigate the ability of other whiskers-body parts to evoke the same recorded neurons to characterize their receptive fields. Although such information would have been useful, it was not the aim of the present study to elucidate the specific role of these recorded neurons in processing whisker A2 information and thus requires additional investigation that is beyond the scope of the present study.

In an additional animal, imaging of the whisker E2 functional representation was similarly followed by single-unit recording. In line with our findings described above for whisker A2, evoked single units in both the supragranular and granular layers were present beyond the PMBSF at a distance of up to $1.45 \mathrm{~mm}$ from the center of the E2 barrel, located near, if not within, the trunk region of SI. There was also a similar tendency for an increase in latency and a decrease in magnitude of evoked single-unit responses with increasing distance from the E2 barrel.

\section{DISCUSSION}

We found several similarities between centrally located and border whisker functional representations. Besides replicating our previous findings of a large functional representation for a centrally located whisker relative to the appropriate barrel (ChenBee and Frostig, 1996; Chen-Bee et al., 1996; Masino and Frostig, 1996; Prakash et al., 1996; Polley et al., 1999a,b), we extended these findings to the border whiskers A2 and E2. Furthermore, for both centrally located and border whiskers, evoked optical and spiking activity is strongest above the appropriate barrel and diminishes progressively in strength away from the barrel in all directions along the tangential plane. These findings suggest that a large, tangential spread of activity can be evoked by any single whisker and is a basic functional feature of the rat whisker-tobarrel system.

Our finding of a large functional representation for a centrally located whisker confirms previous reports that stimulation of a centrally located whisker can evoke activity beyond its appropriate barrel along the tangential plane. Although subthreshold neuronal activity is thought to be a component of the evoked activity captured with intrinsic signal imaging (Das and Gilbert, 1995; Toth et al., 1996), evidence from our laboratory (Polley et al., 1999a) and others (Chapin, 1986; Armstrong-James and Fox, 1987; Peterson et al., 1998; Ghazanfar and Nicolelis, 1999; Petersen and Diamond, 2000) support the existence of suprathreshold activity contributing to the large functional representation of a whisker. Intracortical projections are thought to provide one anatomical substrate by which a centrally located whisker can evoke activity at far distances from either its appropriate barrel (Fox, 1994) (but see Goldreich et al., 1999) or, more likely, from cells in the supragranular or infragranular layers that reside above or below the appropriate barrel (the barrel column). Other sources that could potentially contribute to such spread include subcortical projections and feedback connections from higher 

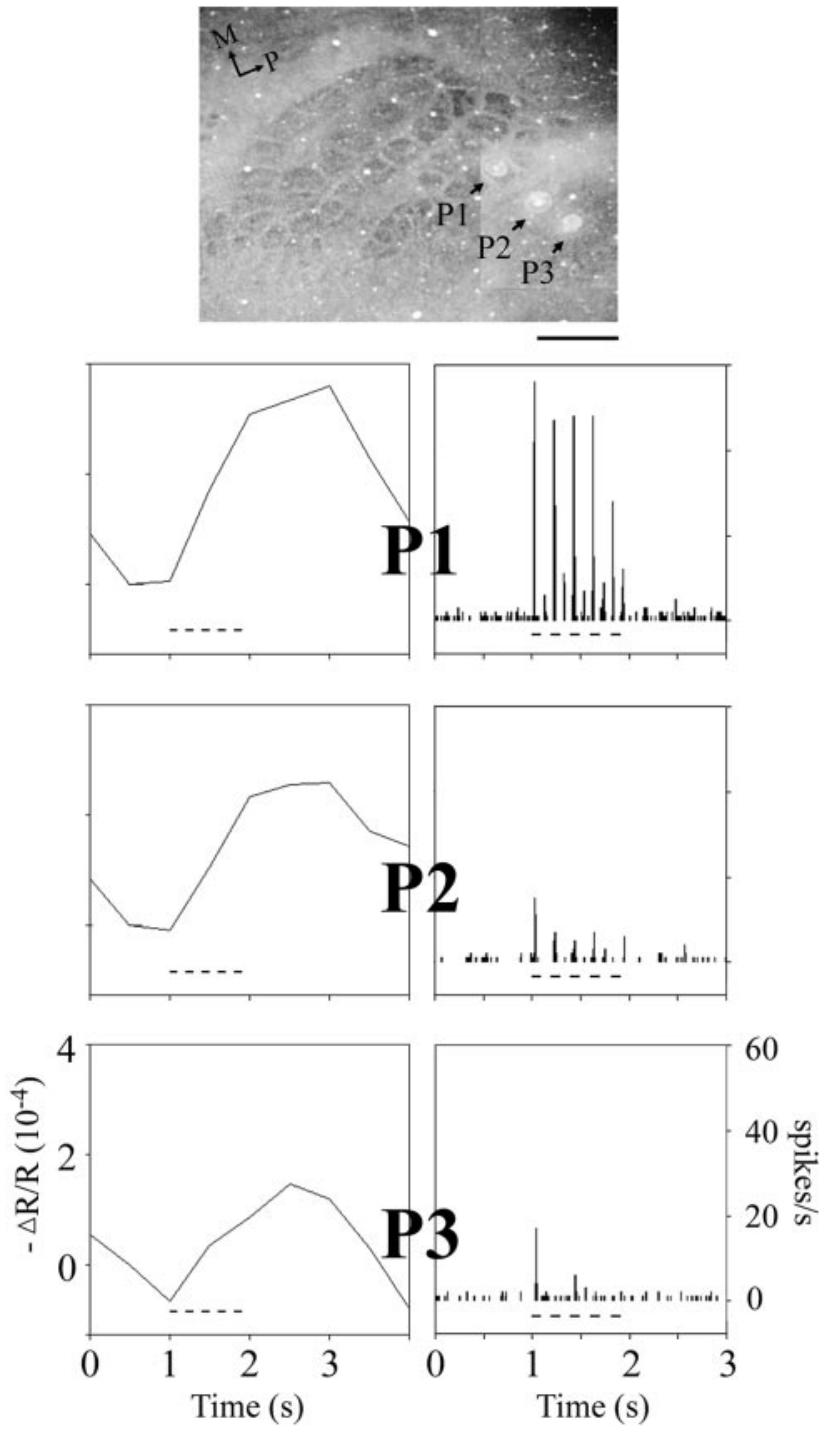

Figure 6. Single-unit responses evoked by whisker A2 stimulation are present outside the PMBSF in neighboring dysgranular zones. Top panel, Electrode penetrations of a representative animal were aligned with the location of the A2 barrel (P1) and at two additional locations of increasing distance away from the A2 barrel $(P 2, P 3)$, as verified with electrolytic lesions in layer IV of the PMBSF stained with CO. Scale bar, $1 \mathrm{~mm} . M$, Medial; $P$, posterior. Examples of PSTHs are provided for single cells recorded in the supragranular layer (right column) to illustrate that the magnitude of single-unit response is strongest above the A2 barrel and decreases in strength with distance from the A2 barrel along the tangential plane. Discrete sampling $\left(0.01 \mathrm{~mm}^{2}\right)$ of intrinsic signals overlying the same locations as the electrode penetrations (left column) exhibit similar properties as single-unit responses in that the magnitude of intrinsic signal response also peaks above the A2 barrel and decays in magnitude over distance. The five dashes near the $x$-axes depict the delivery of whisker stimulation.

cortical areas. A potential source particularly relevant to ISI is the scattering of light and its contribution to the large spread of evoked IS activity captured by the imaging camera. However, we verified previously that the large spread of IS activity evoked by a central whisker has underlying evoked single-unit responses (Polley et al., 1999a), and here we provide a similar verification for the border whisker A2.

In the present study, for the first time, we characterize the tangential spread of activity evoked by whisker A2 whose barrel is located at the posterolateral border of the PMBSF. We find that whisker A2 functional representation as assessed with intrinsic signal imaging extends beyond the columnar border of the PMBSF into the surrounding DZ, with underlying suprathreshold single-unit activity in both the supragranular and granular depths of this zone. Our results extend significantly the region of the DZ in which single whisker responses may be recorded. Although the activity evoked by the border whisker A2 may reach the DZ via projections from the PMBSF coursing through the white matter, this possibility is unlikely for three reasons. First, there is our observed lack of an obvious transition in evoked imaging activity between the PMBSF and the DZ. Second, there is also our observed similarity between the representations of the border whisker A2 and the centrally located whisker C2 with respect to the decay patterns of the amplitude of evoked intrinsic signals and single units at increasing distances away from the appropriate barrel. Third, similarly large representations that decayed smoothly away from peak activity were obtained for another border whisker, E2, as well as other whiskers such as $\alpha$, $\beta$, SO. These observations suggest the involvement of a more local anatomical framework underlying the large functional representation of any whisker, including border whiskers. Preliminary results using discrete biocytin tracer injections hint at the existence of such local intracortical projections extending away from above and below the appropriate barrel of border whiskers A2 and E2 in all directions, including outside the PMBSF into the DZ zone (our unpublished observations) and is consistent with results reported by Kim and Ebner (1999) for central whiskers. Hence, combined with the large functional representations observed for various border whiskers, our results suggest that, as with a centrally located whisker, the functional representation of any border whisker is large, with underlying suprathreshold activity of a border whisker propagating outside of the PMBSF, possibly along intracortical projections.

\section{General implications}

Because a whisker occupies a small, point-like area on the somatosensory epithelium, the large functional representation of a whisker may also be referred to as the cortical point spread of that whisker. We found that the cortical point spread of different whiskers is similar in size despite differences in structural characteristics of the whiskers themselves, such as their length and thickness, and differences between the size of the barrels of the whiskers (Fig. 4B), with the latter finding suggesting that the spatial spread of primary thalamocortical projections to the cortex (as estimated with $\mathrm{CO}$ ) need not predict the spatial spread of evoked activity (as assessed with ISI and single-unit recording). Finally, the size of the cortical point spread of these whiskers is similar despite the fact that two of the representations of the whiskers, E2 and A2, are located at the opposite borders of the PMBSF: A2 is near AI, whereas E2 is near the rest of the body map in SI. Therefore, our findings suggest that a large cortical point spread is a basic functional feature of the rat whisker-tobarrel system and raises the question of whether it may also be a functional feature of primary sensory cortex in general.

In the primary auditory cortex of the rat, guinea pig, and cat, a pure tone, which may be considered a point-like stimulus for the auditory system, has been found previously to evoke a large area of activity, as assessed with imaging of intrinsic signals or voltagesensitive dyes and/or single-unit recordings (Taniguchi and Nasu, 1993; Uno et al., 1993; Phillips et al., 1995; Bakin et al., 1996; Hess and Scheich, 1996). In the cat and monkey, a small, point-like 
Table 1. Response properties of neurons, evoked by whisker A2 stimulation, at increasing tangential distances from the whisker A2 barrel

\begin{tabular}{|c|c|c|c|c|}
\hline \multirow[b]{2}{*}{ Depth } & \multirow[b]{2}{*}{ Measure } & \multicolumn{3}{|c|}{ Average distance from peak } \\
\hline & & P1 (Peak) & $\mathrm{P} 2(570 \mu \mathrm{m})$ & P3 $(1050 \mu \mathrm{m})$ \\
\hline \multicolumn{5}{|l|}{ Supragranular $(300-400 \mu \mathrm{m})$} \\
\hline \multicolumn{5}{|l|}{ \# Responsive/\# recorded } \\
\hline Animals & & $4 / 4$ & $4 / 4$ & $1 / 4$ \\
\hline Cells & & $10 / 10$ & $10 / 11$ & $3 / 12$ \\
\hline Mean evoked latency (msec) & & 11.25 & 19.75 & 30.33 \\
\hline SE evoked latency (msec) & & 0.55 & 2.48 & NA \\
\hline Mean spontaneous magnitude (spikes/sec) & & 1.39 & 1.13 & 1.5 \\
\hline SE spontaneous magnitude (spikes/sec) & & 0.58 & 0.44 & NA \\
\hline Mean evoked magnitude (spikes/sec) & & 54.86 & 35.12 & 38.50 \\
\hline SE evoked magnitude (spikes/sec) & & 17.39 & 3.16 & NA \\
\hline \multicolumn{5}{|l|}{ Granular $(600-700 \mu \mathrm{m})$} \\
\hline \multicolumn{5}{|l|}{ \# Responsive/\# recorded } \\
\hline Animals & & $4 / 4$ & $4 / 4$ & $1 / 4$ \\
\hline Cells & & $8 / 11$ & $10 / 12$ & $1 / 12$ \\
\hline Mean evoked latency (msec) & & 7.95 & 15.25 & 34.00 \\
\hline SE mean evoked latency (msec) & & 0.38 & 1.53 & NA \\
\hline Mean spontaneous magnitude (spikes/sec) & & 4.38 & 2.97 & 3.1 \\
\hline SE spontaneous magnitude (spikes/sec) & & 0.52 & 0.99 & NA \\
\hline Mean evoked magnitude (spikes/sec) & & 68.54 & 48.20 & 36.90 \\
\hline SE evoked magnitude (spikes/sec) & & 9.81 & 3.31 & NA \\
\hline
\end{tabular}

P1 is aligned with the whisker A2 barrel, whereas P2 and P3 are located in the dysgranular zone neighboring the primary somatosensory cortex.

visual stimulus has also been found to evoke a very large area of activity in primary visual cortex, as detected with imaging of voltage-sensitive dyes or intrinsic signals or intracellular recording (Grinvald et al., 1994; Das and Gilbert, 1995; Toth et al., 1996; Bringuier et al., 1999). Similar to the rat PMBSF, spiking (i.e., suprathreshold) activity can be found over most of the cortical point spread in the rat AI (Bakin et al., 1996), although contribution from subthreshold activity may also be present in both rat PMBSF and AI. In contrast to the rat somatosensory and auditory cortices, suprathreshold activity colocalizes with the central region of the cortical point spread in the primary visual cortex of the cat and monkey, whereas subthreshold activity colocalizes with the remaining peripheral regions (Grinvald et al., 1994; Das and Gilbert, 1995; Toth et al., 1996; Bringuier et al., 1999). Regardless of species or sensory cortex, intracortical projections have been suggested as a possible anatomical substrate underlying the large cortical point spread in all of these studies. Together, these results suggest that activation of a large volume of primary sensory cortex by a point stimulus is a basic functional feature preserved across different cortical modalities and species.

Our results have another implication. Although we report here that the point spread of a border whisker can extend smoothly into the DZ neighboring the posterolateral border of the PMBSF, we obtained preliminary results suggesting that high-frequency pure tones can evoke large point spreads extending smoothly outside the anteromedial border of the AI into the same DZ (B. Brett-Green, J. Curley, C. H. Chen-Bee, and R. D. Frostig, unpublished observations). Such large functional representations observed specifically for both border whiskers and border pure tones, an observation that revisits once again the theme of large cortical point spreads as a basic functional property of the cortex, introduce the opportunity for overlap between whisker and pure tone representations. This potential overlap would suggest that the DZ separating primary somatosensory and auditory cortex may contain a polysensory cortical region, as has been indicated in previous evoked potential studies (Barth et al., 1993; Di et al., 1994). A multimodal overlap along a 1.0-mm-wide cortical strip between SI and primary motor cortex (MI) has been reported previously along the hindpaw and part of the forepaw representation area of SI and MI of the rat (Hall and Lindholm, 1974; Sapienza et al., 1981; Donoghue and Wise, 1982; Sanderson et al., 1983; Chapin and Woodward, 1986). Although offering the opportunity of overlap between different sensory modalities and therefore the opportunity for polysensory integration, the largeness of functional representations has its limitations and thus would also place constraints on the amount and nature of polysensory integration. For example, we observed that whisker A2 but not whisker E2 or C2 can evoke activity in the DZ separating the primary somatosensory and auditory cortex, suggesting that this potential polysensory region would respond only to certain whiskers, specifically those whiskers whose associated barrels are located near the posterolateral border of the PMBSF. Likewise, this potential polysensory region may only respond to those pure tones represented near the anteromedial border of AI.

Because there is no a priori reason for restricting polysensory integration of information to only a subset of whiskers and a subset of pure tones, we find intriguing the possibility that the potential overlap between border whisker and border pure tone point spreads need not indicate the presence of polysensory integration. The potential, multiple interpretations of this overlap between border whisker and border pure tone point spreads, i.e., indicative versus not indicative of multimodal processing, serve as an example of the challenges faced by researchers in search of a satisfactory definition of the functional separation between different modalities in primary cortex. 


\section{REFERENCES}

Abeles M (1982) Quantification, smoothing, and confidence limits for single-units' histograms. J Neurosci Methods 5:317-325.

Akers RM, Killackey HP (1978) Organization of corticocortical connections in the parietal cortex of the rat. J Comp Neurol 181:513-537.

Armstrong-James M, Fox K (1987) Spatiotemporal convergence and divergence in the rat S1 "barrel" cortex. J Comp Neurol 263:265-281.

Armstrong-James M, Callahan CA, Friedman MA (1991) Thalamocortical processing of vibrissal information in the rat. I. Intracortical origins of surround but not centre-receptive fields of layer IV neurones in the rat S1 barrel field cortex. J Comp Neurol 303:193-210.

Armstrong-James M, Fox K, Das-Gupta A (1992) Flow of excitation within rat barrel cortex on striking a single vibrissa. J Neurophysiol 68:1345-1358

Bakin JS, Kwon MC, Masino SA, Weinberger NM, Frostig RD (1996) Suprathreshold auditory cortex activation visualized by intrinsic signal optical imaging. Cereb Cortex 6:120-130.

Barth DS, Kithas J, Di S (1993) Anatomic organization of evoked potentials in rat parietotemporal cortex: somatosensory and auditory responses. J Neurophysiol 69:1837-1849.

Bringuier V, Chavane F, Glaeser L, Fregnac Y (1999) Horizontal propagation of visual activity in the synaptic integration field of area 17 neurons. Science 283:695-699.

Carvell GE, Simons DJ (1987) Thalamic and corticocortical connections of the second somatic sensory area of the mouse. J Comp Neurol 265:409-427.

Catalano SM, Robertson RT, Killackey HP (1996) Individual axon morphology and thalamocortical topography in developing rat somatosensory cortex. J Comp Neurol 367:36-53.

Chapin JK (1986) Laminar differences in sizes, shapes, and response profiles of cutaneous receptive fields in the rat SI cortex. Exp Brain Res 62:549-559.

Chapin JK, Lin CS (1984) Mapping the body representation in the SI cortex of anesthetized and awake rats. J Comp Neurol 229:199-213.

Chapin JK, Woodward DJ (1986) Distribution of somatic sensory and active-movement neuronal discharge properties in the MI-SI cortical border area in the rat. Exp Neurol 91:502-523.

Chapin JK, Sadeq M, Guise JL (1987) Corticocortical connections within the primary somatosensory cortex of the rat. J Comp Neurol 263:326-346.

Chen-Bee CH, Frostig RD (1996) Variability and interhemispheric asymmetry of single-whisker functional representations in rat barrel cortex. J Neurophysiol 76:884-894.

Chen-Bee CH, Kwon MC, Masino SA, Frostig RD (1996) Areal extent quantification of functional representations using intrinsic signal optical imaging. J Neurosci Methods 68:27-37.

Chen-Bee CH, Polley DB, Brett-Green B, Prakash N, Kwon MC, Frostig RD (2000) Visualizing and quantifying evoked cortical activity assessed with intrinsic signal imaging. J Neurosci Methods 97:157-173.

Das A, Gilbert CD (1995) Long-range horizontal connections and their role in cortical reorganization revealed by optical recording of cat primary visual cortex. Nature 375:780-784

Di S, Brett B, Barth DS (1994) Polysensory evoked potentials in rat parietotemporal cortex: combined auditory and somatosensory responses. Brain Res 642:267-280.

Donaldson L, Hand PJ, Morrison AR (1975) Cortico-thalamic relationships in the rat. Exp Neurol 47:448-458.

Donoghue JP, Wise SP (1982) The motor cortex of the rat: cytoarchitecture and microstimulation mapping. J Comp Neurol 212:76-88.

Fabri M, Burton H (1991) Ipsilateral cortical connections of primary somatic sensory cortex in rats. J Comp Neurol 311:405-424.

Fox K (1992) A critical period for experience-dependent synaptic plasticity in rat barrel cortex. J Neurosci 12:1826-1838.

Fox K (1994) The cortical component of experience-dependent synaptic plasticity in the rat barrel cortex. J Neurosci 14:7665-7679.

Frostig RD, Lieke EE, Ts'o DY, Grinvald A (1990) Cortical functional architecture and local coupling between neuronal activity and the microcirculation revealed by in vivo high-resolution optical imaging of intrinsic signals. Proc Natl Acad Sci USA 87:6082-6086.

Ghazanfar AA, Nicolelis MA (1999) Spatiotemporal properties of layer $\mathrm{V}$ neurons of the rat primary somatosensory cortex. Cereb Cortex 9:348-361.

Goldreich D, Kyriazi HT, Simons DJ (1999) Functional independence of layer IV barrels in rodent somatosensory cortex. J Neurophysiol 82:1311-1316.

Gottlieb JP, Keller A (1997) Intrinsic circuitry and physiological properties of pyramidal neurons in rat barrel cortex. Exp Brain Res 115:47-60.

Grinvald A, Lieke E, Frostig RD, Gilbert CD, Wiesel TN (1986) Functional architecture of cortex revealed by optical imaging of intrinsic signals. Nature 324:361-364.

Grinvald A, Lieke EE, Frostig RD, Hildesheim R (1994) Cortical pointspread function and long-range lateral interactions revealed by real- time optical imaging of macaque monkey primary visual cortex. J Neurosci 14:2545-2568.

Hall RD, Lindholm EP (1974) Organization of motor and somatosensory neocortex in the albino rat. Brain Res 66:23-38.

Hess A, Scheich H (1996) Optical and FDG mapping of frequencyspecific activity in auditory cortex. NeuroReport 7:2643-2647.

Hodge Jr CJ, Stevens RT, Newman H, Merola J, Chu C (1997) Identification of functioning cortex using cortical optical imaging. Neurosurgery 41:1137-1145.

Hoeflinger BF, Bennett-Clarke CA, Chiaia NL, Killackey HP, Rhoades RW (1995) Patterning of local intracortical projections within the vibrissae representation of rat primary somatosensory cortex. J Comp Neurol 354:551-563.

Jensen KF, Killackey HP (1987) Terminal arbors of axons projecting to the somatosensory cortex of the adult rat. I. The normal morphology of specific thalamocortical afferents. J Neurosci 7:3529-3543.

Killackey HP (1973) Anatomical evidence for cortical subdivisions based on vertically discrete thalamic projections from the ventral posterior nucleus to cortical barrels in the rat. Brain Res 51:326-331.

Killackey HP, Leshin S (1975) The synaptic organization of specific thalamocortical projections to the posteromedial barrel subfield of the rat somatic sensory cortex. Brain Res 86:469-472.

Killackey HP, Belford G, Ryugo R, Ryugo DK (1976) Anomalous organization of thalamocortical projections consequent to vibrissae removal in the newborn rat and mouse. Brain Res 104:309-315.

Kim U, Ebner FF (1999) Barrels and septa: separate circuits in rat barrels field cortex. J Comp Neurol 408:489-505.

Kleinfeld D, Delaney KR (1996) Distributed representation of vibrissa movement in the upper layers of somatosensory cortex revealed with voltage-sensitive dyes. J Comp Neurol 375:89-108.

Koralek KA, Jensen KF, Killackey HP (1988) Evidence for two complementary patterns of thalamic input to the rat somatosensory cortex. Brain Res 463:346-351.

Koralek KA, Olavarria J, Killackey HP (1990) Areal and laminar organization of corticocortical projections in the rat somatosensory cortex. J Comp Neurol 299:133-150.

Land PW, Simons DJ (1985) Cytochrome oxidase staining in the rat SmI barrel cortex. J Comp Neurol 238:225-235.

Land PW, Buffer SA, Yaskowska JD (1995) Barreloids in the adult rat thalamus: three-dimensional architecture and relationship to somatosensory cortical barrels. J Comp Neurol 355:573-588.

Lu SM, Lin RC (1993) Thalamic afferents of the rat barrel cortex: a light- and electron-microscopic study using Phaseolus vulgaris leucoagglutinin as an anterograde tracer. Somatosens Mot Res 10:1-16.

Masino SA, Frostig RD (1996) Quantitative long-term imaging of the functional representation of a whisker in rat barrel cortex. Proc Nat Acad Sci USA 93:4942-4947.

Masino SA, Kwon MC, Dory Y, Frostig RD (1993) Characterization of functional organization within rat barrel cortex using intrinsic signal optical imaging through a thinned skull. Proc Natl Acad Sci USA 90:9998-10002.

Narayan SM, Santori EM, Blood AJ, Burton JS, Toga AW (1994) Imaging optical reflectance in rodent barrel and forelimb sensory cortex. NeuroImage 1:181-190.

Olavarria J, Van Sluyters RC, Killackey HP (1984) Evidence for the complementary organization of callosal and thalamic connections within rat somatosensory cortex. Brain Res 291:364-368.

Orbach HS, Cohen LB, Grinvald A (1985) Optical mapping of electrical activity in rat somatosensory and visual cortex. J Neurosci 5:1886-1895.

Petersen RS, Diamond ME (2000) Spatial-temporal distribution of whisker-evoked activity in rat somatosensory cortex and the coding of stimulus location. J Neurosci 20:6135-6143.

Peterson BE, Goldreich D, Merzenich MM (1998) Optical imaging and electrophysiology of rat barrel cortex. I. Responses to small singlevibrissa deflections. Cereb Cortex 8:173-183.

Phillips DP, Semple MN, Kitzes LM (1995) Factors shaping the tone level sensitivity of single neurons in posterior field of cat auditory cortex. J Neurophysiol 73:674-686.

Polley DB, Chen-Bee CH, Frostig RD (1999a) Two directions of plasticity in the sensory-deprived adult cortex. Neuron 24:623-637.

Polley DB, Chen-Bee CH, Frostig RD (1999b) Varying the degree of single-whisker stimulation differentially affects phases of intrinsic signals in rat barrel cortex. J Neurophysiol 81:692-701.

Prakash N, Cohen-Cory S, Frostig RD (1996) Rapid and opposite effects of BDNF and NGF on the functional organization of the adult cortex in vivo. Nature 381:702-706.

Sanderson KJ, Welker W, Shambes GM (1983) Reevaluation of motor cortex and of sensorimotor overlap in cerebral cortex of albino rats. Brain Res 292:251-260.

Sapienza S, Talbi B, Jacquemin J, Albe-Fessard D (1981) Relationship between input and output of cells in motor and somatosensory cortices of the chronic awake rat: a study using glass micropipettes. Exp Brain Res 43:47-56. 
Saporta S, Kruger L (1977) The organization of thalamocortical relay neurons in the rat ventrobasal complex studied by the retrograde transport of horseradish peroxidase. J Comp Neurol 174:187-208.

Schroder R, Luhmann HJ (1997) Morphology, electrophysiology and pathophysiology of supragranular neurons in rat primary somatosensory cortex. Eur J Neurosci 9:163-176.

Sheth BR, Moore CI, Sur M (1998) Temporal modulation of spatial borders in rat barrel cortex. J Neurophysiol 79:464-470.

Simons DJ, Carvell GE, Hershey AE, Bryant DP (1992) Responses of barrel cortex neurons in awake rats and effects of urethane anesthesia. Exp Brain Res 91:259-272.

Taniguchi I, Nasu M (1993) Spatio-temporal representation of sound intensity in the guinea pig auditory cortex observed by optical recording. Neurosci Lett 151:178-181.

Toth LJ, Rao SC, Kim DS, Somers D, Sur M (1996) Subthreshold facilitation and suppression in primary visual cortex revealed by intrinsic signal imaging. Proc Natl Acad Sci USA 93:9869-9874.

Ts'o DY, Frostig RD, Lieke EE, Grinvald A (1990) Functional organization of primate visual cortex revealed by high resolution optical imaging. Science 249:417-420.

Uno H, Murai N, Fukunishi K (1993) The tonotopic representation in the auditory cortex of the guinea pig with optical recording. Neurosci Lett 150:179-182.

Wallace MN (1987) Histochemical demonstration of sensory maps in the rat and mouse cerebral cortex. Brain Res 418:178-182.

Welker C, Sinha MM (1972) Somatotopic organization of SmII cerebral neocortex of the albino rat. Brain Res 37:132-136.

Welker C, Woolsey TA (1974) Structure of layer IV in the somatosensory neocortex of the rat: description and comparison with the mouse. J Comp Neurol 158:437-453.

Wise SP, Jones EG (1978) Developmental studies of thalamocortical and commissural connections in the rat somatic sensory cortex. J Comp Neurol 178:187-208.

Wong-Riley MT, Welt C (1980) Histochemical changes in cytochrome oxidase of cortical barrels after vibrissal removal in neonatal and adult mice. Proc Natl Acad Sci USA 77:2333-2337.

Woolsey TA, Van der Loos H (1970) The structural organization of layer IV in the somatosensory region (SI) of mouse cerebral cortex. The description of a cortical field composed of discrete cytoarchitectonic units. Brain Res 17:205-242.

Woolsey TA, Dierker ML, Wann DF (1975) Mouse SmI cortex: qualitative and quantitative classification of golgi-impregnated barrel neurons. Proc Natl Acad Sci USA 72:2165-2169. 